\title{
Analysis of the No Return Point Hypothesis: The Effect of Audio and Visual Stimuli in the Fast Movements Inhibition
}

\author{
${ }^{1}$ Ali Kheyrandish, ${ }^{1}$ Alireza Saberi Kakhki ${ }^{*}{ }^{1}$ Hamidreza Taheri \\ ${ }^{1}$ Department of Motor Behavior, Faculty of Sports Science, Ferdowsi University of Mashhad, Mashhad, \\ Iran.
}

Submitted 01 June 2020; Accepted in final form 07 September 2020.

\begin{abstract}
Background. The No Return Point hypothesis is one of the research areas that has been done in line with the motor program. In this hypothesis emphasized an inability to inhibition move after its start by the motor program. Several factors are affecting the mechanism of this inhibition. Objectives. In this study, we investigate the effects of audio and visual stimuli on blocking quick moves to test the No Return Point hypothesis. In the final part of the study, we explore the effects of the assignment based on tests conducted by Slater \& Hammel, who first began to test this hypothesis. Methods. In this study, 40 male participants (age 23-29) were selected and organized in four different groups, i.e., digital- visual stimulus, an auditory stimulus, visual and auditory stimuli, and visual stimulus with an analog pattern that simulated the Slater and Hamel's experiment. Each of these groups practiced different stop-signal and did a retention test. We used RMSE for statistical analysis. Results. The results showed that the reaction speed of the audio group is considerably faster than other groups ( $\mathrm{sig}=0.00028, \mathrm{p}<0.05$ ). It was also found that the motor program is active in a period before and after the target (stop) time, and it seems that this process is independent of the type of stimuli and assignment $(\mathrm{p}<0.05)$. Besides, it was found that the reported time for Slater and Hamel test is $210 \mathrm{MS}$ in this experiment. In other words, participants were not able to stop motion for the announced stop time for around 210 Ms.; however, it was not observed for all the groups. Conclusion. Based on the result, participants were significantly dependent on the assignment $(\mathrm{p}<0.05)$. Moreover, this dependency can affect the result. It seems in that study time providing achieved relying on the types of tasks used, and the type of the variable used to measure the statistical have been impressive on the results. Thus, the results of the Slater and Hamel experiments should be analyzed with caution. It seems that the announced time in that research (Slater and Hemel) is based on the employed assignment type, and the variable type for statistical analysis was influencing the results. Thus, not only the motion stop time in response to the stop stimuli is different, but also these times are significantly dependent on the stimuli and test condition.
\end{abstract}

KEYWORDS: No Return Point Hypothesis, Audio-Visual Stimuli, Fast Movements Inhibition.

\section{INTRODUCTION}

Since the very first academic studies in the field of motor control, there were many questions regarding the control of fast movements (1). By presenting several hypotheses, researchers have tried to answer these questions. To clarify the subject, they categorized the stimulus to study them in detail and more precise. In one of these classifications, movements were divided into two groups, fast and slow. Fast movements are those, which the person does not have enough time to act, while in the slow stimulus, he has plenty of time (2).

Schmidt's theory was used as an explanation for the motor controls for years, and it was

*. Corresponding Author:

Alireza Saberi Kakhki, Associate Professor

E-mail: askakhki@um.ac.ir 
accepted later as the Generalized Motor Program (GMP) (1). Many experiments were conducted in agreement with this hypothesis (3). These Laboratory experiments tried to transfer the motor program from a virtual state into a more objective form.

Studies on how to control rapid movements are generally performed in a laboratory and controlled setting. One of the common patterns in designing studies in this field is the go-stop and inhibition pattern. The No Return Point hypothesis is one of the concepts used in studies of movement inhibition and the study of mechanisms in movement stop. This hypothesis states that when a movement begins, it is impossible to stop the movement produced for a specific time (approximately $110 \mathrm{msec}$ ). Schmidt has used the No Return Point hypothesis in his theory (GMP Theory) (4). He explained that when a motor action was programmed and sent towards the muscles, it is impossible to stop or reschedule it within a specific time frame. The No Return Point hypothesis was tested by Slater \& Hammel (5) in laboratory conditions, and it confirmed that hypothesis. This hypothesis was improved over time (6). Since many theories, including Schmidt's theory of motor program, use the concepts of movement inhibition and No Return Point, it is necessary to study results in this field to benefit from its concepts. Some explanations had been presented to interpret the results. For example, it was said that a totality performs the movement as a whole or non after preparation. Any variations in this totality structure are practically impossible before the end of the action (7). This phenomenon was explained by Schmidt (8) and the motor program(MP) theory in a different way later.

Regarding this matter, it was stated that before starting the action, the motor program plans, programs, and develops the movement. It is impossible to stop the movement within a specific timeframe after it starts. The open-loop control theory explaining the fast movements based on motor program, states that the motor program monitors and organizes action's parts during the fast movements. As explained, the theory of motor program and the hypothesis use the same literature to study fast motion. Some of these studies are as follow: Schmidt's Schema Theory $(8,9)$, The Trigger or Jockey theory $(10)$, and Henry-Harrison's theory (6). Almost in all of these studies, the motor program's concept and inability to stop after the start point can be seen. The motor program theory has an apprehensible virtual structure that researchers can use to explain how movement is doing.

On the other hand, critics try to note the contradictions in this theory. Schmidt has used other explanations to confirm the motor program's structure $(1,8,11,12)$. These explanations were inspired from Henry and Harrison's work about the No Return Point hypothesis.

Various studies have been conducted in the conformation of the No Return Point hypothesis, which most were in laboratory conditions (1319). Gao and Zelaznik (20) evaluated Henry and Harrison's hypothesis in detail. They showed that the task type, the scaling, and the data analysis methods for the motor pattern are affecting the results. Based on this study, the re-analysis of the data indicated that the time presented by the Henry and Harrison experiments not consistent with extending to different people. Also, some studies questioned the task types which were used in Henry and Harrison's experiment, because due to the bisectional nature of the movement that is the Reaction time (RT) and movement time (MT), people use various strategies in order to reach the objective point, which could affect the results (9, 20, 21). As mentioned earlier, various stopping times have been directly or indirectly stated in some studies $(14,17,22)$. Over the following years, it was seen in some studies that the No Return Point had not been constant in some experiments, and the participant was able to stop his action after starting it rapidly (23). Also, the time stated in some studies was not compatible with the 110 milliseconds mentioned in the Slater \& Hammel experiment $(13,14,20,21)$. In similar studies, the effect of other variables was analyzed, which include the individual differences $(9,22$, 24-26) and neurodevelopmental disabilities (27), which produce different results. In a review of how these results emerge, motor complexity can be mentioned as the most important one $(15,28$, 29). In some studies that were performed later based on re-analysis of the confirming studies like Henry and Harrison's one, it was determined that this subject has complexity and presented analysis of the motor program as an explanation of the No Return Point theory's result, may not be accepted(20). Also the used task and data analysis 
method in some studies have been evaluated again and the results were slightly changed in some of them.

Investigations into movement inhibition studies have shown that some factors are useful in recording stop movement time and No Return Point. The time recorded in the Slater and Hummel experiment (110 milliseconds) was performed under laboratory conditions and on some specific variables. Different inhibition times in experiments in this field may be due to some intervening variables in these experiments. Therefore, re-examining the Slater and Hummel test and controlling the variables involved can help clarify the factors influencing the registration of the point of inhibition and no return. In some studies which most have been concluded recently, the reaction time differences causing by a different stimulus have been pointed out (6). It has been reported that the type of stimulus can change the simple reaction time. In studies that have directly or indirectly reported the effect of the stimulus on reaction time, it has been seen that the reaction time to an audio stimulus is less than the one caused by a visual one (30).

Reviewing the studies on the No Return Point hypothesis, most of them used visual stimulus as a secondary stimulus. As the reaction time is different for different stimuli, mostly audio and visual, in this study, stimulus type was considered as a significant variable in the No Return Point hypothesis. On the other hand, the closer the research conditions are to the real situation, the more general the results will be (31). On the other hand, it seems that the public's view on the analog clock pattern of Slater and Hammel's experiment persuades them to mostly use the pointer's position in order to respond to the second stimulus. In other words, it seems that people made more use out of spatial predictions. In the present study, it has been tried to examine this hypothesis and use patterns which both prepare the requirements for Slater and Hammel's pattern and limit the people's use of the spatial structure. In this research, by combining hardware and computer software, on the one hand, we try to examine the Slater and Hummel test, on the one hand, to re-evaluate the point hypothesis without return.

On the other hand, we bring the test execution conditions closer to real motion conditions. Investigate the effect of the type of stimulus on the results from a different (auditory) stimulus. In this study, we will examine the difference in results in the new digital model by simulating a hands-on timer pattern.

Therefore, due to these contradictions and the importance of confirming the motor program theory and evaluating the No Return Point hypothesis, it has been tried in this study to control the task type on the one hand, while studying the participants and presented stimulus on the other. This study intends to answer this question: Does the No Return Point change by changing the nature of the presented stimulus and type of tasks?.

\section{MATERIALS AND METHODS}

Participants. Participants were forty male graduate student volunteers(age 23-29). They were aligned to experimental groups based on their simple reaction time.

The experiments were designed similar to Slater and Hammel's pattern and consist of software and hardware of a stopwatch with visual and audio stimuli. Using a hardware Arduino board and LabVIEW (ver 2016) software, we could develop a computer-based code to design software for the experiments. It can be connected to a computer via a USB cable. The Arduino ${ }^{\mathrm{TM}}$ compiler for LabVIEW is a product based on LabVIEW (Laboratory Virtual Instrument Engineering Workbench) by National Instruments. This method can be programmed easily with excellent reliability (32). Unlike Slater and Hummel's pattern that provide a visual stimulus, with this board, it is possible to design a visual and audio stimulus individually or combined. Changing the stop-go time and both analog and digital stopwatch was possible with using this board.

To answer the questions were mentioned earlier, three experiments were designed as follow:

Experiment 1:

In this experiment, the difference between visual, audio, and combined stimuli reaction time was studied to assess whether the stimuli type affects the reaction time (RT) or not. Forty participants answered the visual, audio, and combined stimuli. The task was a simple time reaction. The participant should be sitting in front of a monitor and response to relative stimuli as quickly as possible. When the relative stimulus appeared, he should release the space bottom. For the audio stimulus, the participant should wear a 
headphone, and when he heard the beep, he should release the space bottom. In the third situation, both visual and audio stimuli were employed. The participant should release the space bottom when he saw and heard both stimuli. The reaction time was recorded in an Excel file.

Experiment 2:

This experiment was designed as a response to the generalization of Slater and Hammel's experiment's results based on the stimuli type. The participants were divided into three groups $(\mathrm{N}=9)$ based on their pre-test scores. The training and response pattern was unique for each group.

The first group named visual stimulus (similar to Slater and Hammel experiment), they used the digital stopwatch that could be recorded the time differences. When the movement was started, the digital stopwatch was run, and the participant could see it. The stopwatch was stopped after $1800 \mathrm{msec}$. The participant must release the space bottom when they saw the stop sign. They can see the response time as feedback on display. In the retention stage, it was asked from the participant to do the same task after 48 hours in 5 trials without any feedback. In the next stage, unlike the acquisition stage with $1800 \mathrm{msec}$ pattern, they were asked to respond to a pre-set time (2000, 1950, 1900, 1850, 1540, 1490, 1440, 1390, 1690, 1640,1590 , and $1740 \mathrm{msec}$ ), and this stage was called motion stop. The design pattern of these times was to fulfill not only the assumption of the Slater \& Hammel experiment but also the delay time in motion stop from $60 \mathrm{msec}$ to $410 \mathrm{msec}$ before the start. These times were dispersed in the same frequency in the $1800 \mathrm{msec}$ pattern and was designed randomly.s

The second group was named an audio stimulus, and they have been learned a pattern in the acquisition stage. They hear a beep sound as a start sign through a headset, and after $1800 \mathrm{msec}$, they hear a different beep sound as a stop sign, and they need to release the space bottom. They were told to release the bottom at the same time as stop beep sound, and the difference between the beep sound and their reaction was recorded as response time. The response time was shown as the feedback to try to release the bottom before hearing the sound. In other words, they plan to act before they received the stimulus. In the final stage, like the first group, the sound was played at different stop times, and they must respond when they hear the sound. The response time was monitored. It needs to be mentioned that at this stage, they did not receive any feedback. The next stages were done like the first group.

The third group conducted the combined pattern simultaneously; it means they received both visual and audio stimuli. The acquisition stage was done with the feedback and stopped time's pattern, as mentioned earlier. The response time was recorded as their final score.

In this experiment, the participants conducted 180 trials (60trails $\times 3$ blocks) in the acquisition stage.

Table 1. Groups, Tasks, and Procedure

\begin{tabular}{|c|c|c|c|c|c|c|}
\hline Group & $\begin{array}{c}\text { Number of } \\
\text { Participants }\end{array}$ & Task & $\begin{array}{c}\text { Pre-Test } \\
\text { (trails) }\end{array}$ & $\begin{array}{c}\text { Acquisition } \\
\text { Stop-Time } \\
\end{array}$ & $\begin{array}{l}\text { Retention } \\
\text { Stop-Time }\end{array}$ & $\begin{array}{l}\text { Post-Test } \\
\text { (trails) }\end{array}$ \\
\hline Visual stimulus & 9 & Digital-visual pattern & 5 & $1800 \mathrm{~ms}$ & $\begin{array}{c}1600-1650- \\
1690-1750 \\
\mathrm{~ms}\end{array}$ & 5 \\
\hline Audio stimulus & 9 & $\begin{array}{c}\text { Audio } \\
\text { pattern(headphone) }\end{array}$ & 5 & $1800 \mathrm{~ms}$ & $\begin{array}{c}1600-1650- \\
1690-1750 \\
m s\end{array}$ & 5 \\
\hline $\begin{array}{l}\text { Simultaneous visual } \\
\text { and audio stimulus }\end{array}$ & 9 & $\begin{array}{c}\text { Combine } \\
\text { pattern(visual\&audio) }\end{array}$ & 5 & $1800 \mathrm{~ms}$ & $\begin{array}{c}1600-1650- \\
1690-1750 \\
\mathrm{~ms}\end{array}$ & 5 \\
\hline
\end{tabular}

Experiment 3. In this experiment, we would like to answer whether the used pattern in Slater \& Hammel experiment influenced the results. In this experiment, 20 participants were divided into two groups. In the first group, the participants tried the stopwatch pattern like the visual group in experiment 2 at the acquisition stage. They were tested like that group in retention and transfer stages. The second group was done the same procedure but with an analog stopwatch that consists of an LED as a clock hand that can simulate the digital stopwatch. In this group, the target time was $1800 \mathrm{msec}$, and the transfer time was the same as before. The goal of this 
experiment was to evaluate the author's assumption on the effect of task type on the stop time in the Slater \& Hammel experiment and similar experiments. It seems that the participant was stick to the stopwatch's hand more than focusing on the internal stimulus control process and their prediction was based on the stopwatch's hand's position in a specific time; so this response can have an influence on the main objective of this project which is a determination of no-return point. The results of the digital and analog watch were compared to assess this pattern.

Analysis. Standard deviation was used as raw data for statistical analysis. In this study, the descriptive statistic was used to specify the groups and participants. Data were analyzed by one-way analysis of variance (ANOVA) and analysis of variance with repeated measures. Tukey post hoc test was used to determine the differences. We used SPSS 21 and Excel software for data analysis.

\section{RESULTS}

The results of experiment 1 showed (Table 2) the difference between groups' means is significant $(F=8.323, P=0.00)$. It was showed that the reaction time for audio stimulus is much faster than visual stimulus.

Also, the audio stimulus group was faster than the mixed group, and this difference was significant, but no significant difference was found between the visual stimulus and combined stimulus (Table 3).

The results of the second experiment showed that the participant uses the predicting pattern to decrease their deviation of time recorded. The results of the retention stage also showed that they had learned the presented pattern. In the retention stage, participants were compared with each of the time provided (Table 4). At 1390, 1540, 1800, 1950, and 2000 milliseconds, the difference between the times was significant, and for the other times, there was no significant difference $(\mathrm{p}<0.05)$.

We can stop-time patterns between different groups. We found the disorder between 1960$1850 \mathrm{~ms}$ in every group.

The third experiment results showed (Table 5) that as it was assumed, the results in digital and analog visuals were different. A significant advantage in favor of the visual analog was found $(\mathrm{p}=0.001)$.

Table 2. The Difference Between Groups (Experiment 1)

\begin{tabular}{|l|c|c|c|c|c|c|}
\hline & Sum of Squares & Df & Mean Square & F & p \\
\hline Between groups & 33827.517 & 2 & 16913.758 & 8.238 & 0.000 \\
\hline
\end{tabular}
ANOVA, RT

Table 3. Result of Groups in Tukey HSD Test

\begin{tabular}{lccc}
\hline Group & Mean Difference (I-J) & Std. Error & p \\
\hline Audio & & & 0.103 \\
$\quad$ Visual & -20.85000 & 10.13186 & 0.000 \\
O-V & $-41.12500^{*}$ & 10.13186 & 0.103 \\
Visual & & 10.13186 & 0.116 \\
$\quad$ Audio & 20.85000 & 10.13186 & 0.000 \\
O-V & -20.27500 & 10.13186 & 0.116 \\
O-V & & 10.13186 & \\
Audio & $41.12500^{*}$ & & \\
Visual & 20.27500 & & \\
\hline
\end{tabular}

Multiple Comparisons, Dependent Variable: RT, Tukey HSD

\section{DISCUSSION}

By reviewing research on how to produce and control movements, we are dramatically confronted with the concepts of movement inhibition or patterns of movement redesign. One of the old and fundamental assumptions in this type of study is the No Return Point hypothesis, the principles of which have been used in many types of research in this field $(4,5,14,20,33)$. In this study, by using the results of research in the field of motor control and No Return Point hypothesis and review of research literature, this hypothesis was determined; although much research has been done on this subject is clear, but different inhibition times were recorded $(7,14$, 15). This research was designed using precise practice design methods and with the least interference in the execution of the movement. Its purpose was to investigate various factors such as 
the type of stimulus and the type of task on the motion stop-time pattern and its effects on accepting the No Return Point hypothesis. There has been much research on how to stop movements, each seeking to respond to different dimensions of inhibition and stop movements (21). New test design methods and brain and muscle wave recording devices are standard methods for examining movement stop mechanisms $(13,16)$. However, it should be noted that the use of new and technology-based methods should not take the test conditions out of their normal state. Since the primary purpose of such experiments is to determine the mechanisms of motor control in normal conditions and to use them in sports or rehabilitation, it should be noted that the conditions for conducting this research should be very close to the real conditions. In this study, using computer design capabilities, the initial patterns of experiments related to stopping movements were redesigned and based on this, the no return hypothesis was examined (32). In this study, we tried to use computer hardware and simulator software to design a simple yet precise movement so that the participants could perform the test with the least amount of muscle and mental conflict similar to the conditions of a normal position. The first experiment results showed that audio response time is faster than the visual or combined response times. This result is compatible with Shelton and Kumar study (15, 30). It seems that in the combined sign experiment, the participants focus more on visual stimulus rather than the audio stimulus so it can interpret the difference between combined and audio stimuli.

Table 4. Transfer Stage Result

\begin{tabular}{llccccc}
\hline & \multicolumn{2}{c}{ ANOVA } & & & \\
\hline Task & & Sum of Squares & DF & Mean Square & F & p \\
\hline Transfer 1390 & Between Groups & 380751.462 & 3 & 126917.154 & 3.257 & 0.035 \\
Transfer 1440 & Between Groups & 201757.679 & 3 & 67252.560 & 2.290 & 0.098 \\
Transfer 1490 & Between Groups & 136939.929 & 3 & 45646.643 & 1.157 & 0.342 \\
Transfer 1540 & Between Groups & 342435.445 & 3 & 114145.148 & 2.975 & 0.047 \\
Transfer 1590 & Between Groups & 177233.680 & 3 & 59077.893 & 1.473 & 0.241 \\
Transfer 1640 & Between Groups & 89482.123 & 3 & 29827.374 & .704 & 0.557 \\
Transfer 1690 & Between Groups & 196083.421 & 3 & 65361.140 & 1.408 & 0.259 \\
Transfer 1740 & Between Groups & 445813.788 & 3 & 148604.596 & 2.839 & 0.054 \\
Transfer 1800 & Between Groups & 649528.463 & 3 & 216509.488 & 6.139 & 0.002 \\
Transfer 1850 & Between Groups & 218134.012 & 3 & 72711.337 & 1.845 & 0.160 \\
Transfer 1900 & Between Groups & 431027.636 & 3 & 143675.879 & 2.629 & 0.068 \\
Transfer 1950 & Between Groups & 687029.548 & 3 & 229009.849 & 5.112 & 0.005 \\
Transfer 2000 & Between Groups & 671498.976 & 3 & 223832.992 & 7.564 & 0.001 \\
\hline
\end{tabular}

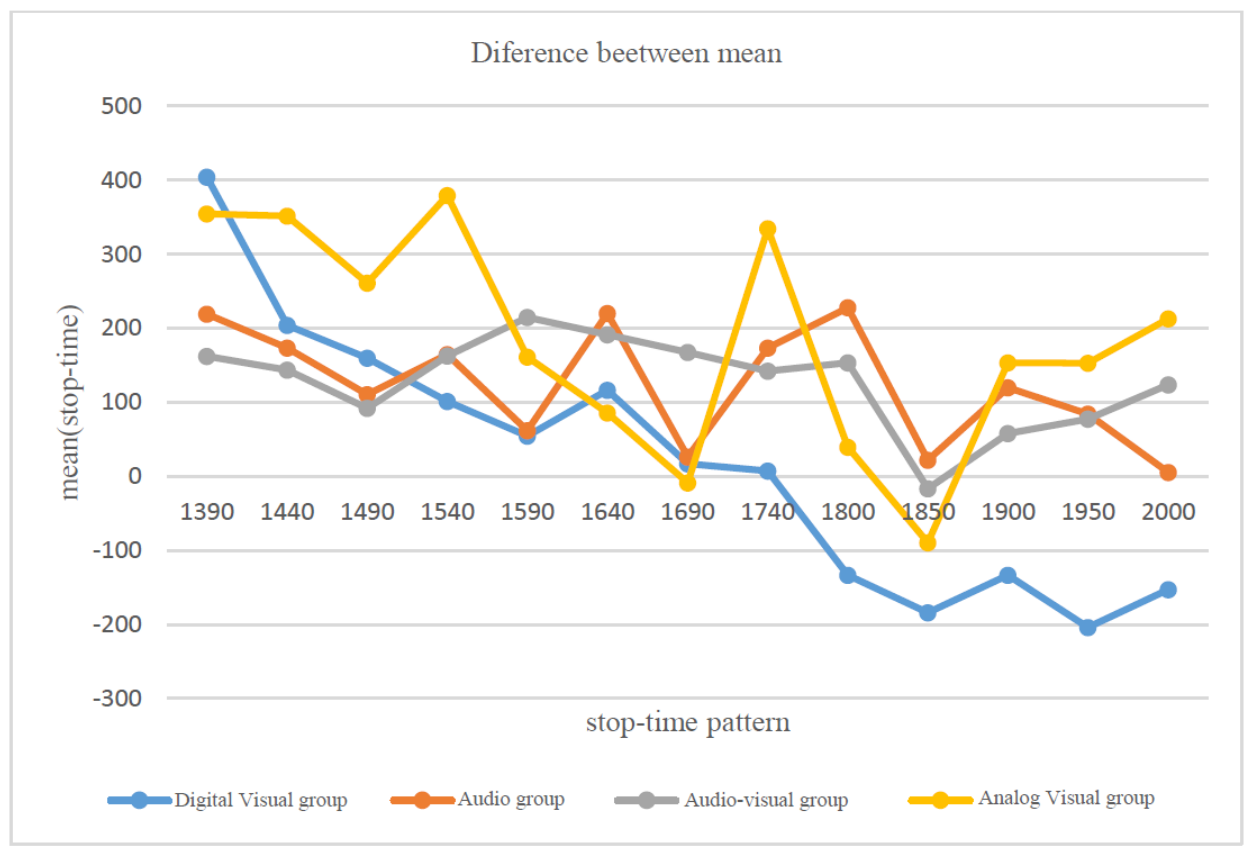

Figure 1. Compare Between Stop-Time in Different Groups. 
Table 5. Different from Digital and Analog Visual Groups

\begin{tabular}{lccc} 
& $\mathbf{F}$ & $\mathbf{d f}$ & $\mathbf{p}$ \\
\hline Equal variances assumed & 2.359 & 13 & 0.001 \\
\hline Independent Samples Test, T-Test for Equality of Means & &
\end{tabular}

In the second experiment, results showed no significant differences between groups in many of times (1590, 1640, 1740, 1850, $1900 \mathrm{msec})$. In other words, the groups behave the same, and the parameters affected by these times are the same. This shows that a motor program is active before and after the target time (stop time), (210msec before and $100 \mathrm{msec}$ after the target time) $(2,16$, 22). It seems that this motor program is independent of stimulus and task type. So as long as this program is active, it is impossible to respond to another stimulus, and if another response happens, it might have interference. Therefore, it seems the time of No Return Point is more extended than Slater-Hummel's report. We propose that time is $210 \mathrm{msec}$.

Nearly this result repeat in the same articles $(14,30)$, but there are many articles at different times (21). The results confirmed the hypotheses of this study based on the effectiveness of the type of stimulus and the type of task and have been repeated in similar studies that have generally examined the effects of visual stimuli and used brainwave recording methods (34). Many studies have suggested a mechanism for controlling movement and activating it before starting to move (35). In some of them, the inability to stop moving has been reported for some time after it started that confirms the existence of a motor program. The present study also confirmed the existence of an abstract mechanism in the production and control of motion. Similar studies have shown that most of these studies report a specific time to inhibit movement, but they are different in each study. It seems that research methods, type of stimulus, research assignments, and some other factors are among the factors that can affect the results in this field $(10,21,26,36$, 37). In most of these studies, it was found that the closer the stopping time was to the start time, the more likely it was to be prevented (14). This confirms the theory of the bottleneck (38). The inability to stop moving for a certain period after the start of the movement is one of the similar cases in such research. Of course, it is impossible to announce a specific time to record a No Return Point, but further research is likely to record a specific period for a No Return Point $(13,14)$. The generalizability of the results of this research should be done with more caution, and in using the results, variables such as the type of task and the related variable should be considered. Also, other variables such as age, gender, and psychological conditions of the subjects may affect the results that the accuracy of this issue should be examined.

Moreover, based on the third experiment results, participants' response to the stop time is highly dependent on task type. This finding does not verify the Slater - Hummel's results. It seems that their results have been influenced by task type and also the used variable of statistical analysis. Our findings, to some extent, confirmed Shelton and Kumar's suggestion (30). Finally, we suggest that a top-down hierarchical mechanism may control intentional behavior, while the participant does not expect the stop time (like 1850 and $1900 \mathrm{msec}$ ), it follows the natural perception process (9).

It is suggested that in order to clarify the various dimensions of the results of a metaanalysis study on the research in this field, the effectiveness of each of the variables should be determined. It seems that from the results of this research, a formula can be obtained that can accurately predict the time of the No Return Point in the patterns of motion inhibition.

\section{CONCLUSION}

This study showed that the stopping time of movements in the motion inhibition pattern is 210 milliseconds. The type of stimulus and the type of task used in this type of research affect the results and change the motion inhibition time. The closer the stop stimulus is to the criterion time, the less likely it is to stop moving.

Although the principles presented in the No Return Point hypothesis have been confirmed, more careful consideration should be given to using the results of such research, and its results should be used with more caution.

\section{APPLICABLE REMARKS}

- Inhibition time can play an essential role in identifying stop and control processes.

- The experimental method used in this research project can be used with great confidence in similar research fields because the results of 
this study are mostly consistent with the results of similar research.

- Researchers should be more careful about using the principle of the No Return Point hypothesis. In laboratory conditions, they should control the type of variables and the type of task. However, the results of this study confirm the basics of this hypothesis.

\section{REFERENCES}

1. Schmidt RA. Control processes in motor skills. Exerc Sport Sci Rev. 1976;4:229-261. pmid: 828577

2. Schmidt RA, Lee TD, Winstein C, Wulf G, Zelaznik HN. Motor control and learning: A behavioral emphasis: Human kinetics2018.

3. Taub E, Berman A. Movement and learning in the absence of sensory feedback. Neuropsychol Spatiall Orient Behav. 1968:173-192.

4. Logan GD, Van Zandt T, Verbruggen F, Wagenmakers EJ. On the ability to inhibit thought and action: general and special theories of an act of control. Psychol Rev. 2014;121(1):66-95. doi: 10.1037/a0035230 pmid: 24490789

5. Slater-Hammel AT. Reliability, accuracy, and refractoriness of a transit reaction. Res Quarter America Associat Health Physic Educat Recreat. 1960;31(2):217-228. doi: 10.1080/10671188.1960.10613098

6. Henry FM, Harrison JS. Refractoriness of a fast movement. Percept Motor Skill. 1961;13(3):351-354. doi: 10.2466/pms.1961.13.3.351

7. Deecke L, Soekadar SR. Beyond the point of no return: Last-minute changes in human motor performance. Proc Natl Acad Sci U S A. 2016;113(21):E2876. doi: 10.1073/pnas.1604257113 pmid: 27147601

8. Schmidt RA, Zelaznik H, Hawkins B, Frank JS, Quinn JrJT. Motor-output variability: a theory for the accuracy of rapid motor acts. Psychol Rev. 1979;86(5):415. doi: 10.1037/0033-295X.86.5.415

9. Rosenbaum DA. Human motor control: Academic press; 2009.

10. Band GP, Van Der Molen MW, Logan GD. Horse-race model simulations of the stop-signal procedure. Acta Psychol. 2003;112(2):105-142. doi: 10.1016/S0001-6918(02)00079-3

11.Schmidt RA. Motor Control and Learning 5th Edition: Human kinetics1988.

12. Smith WM, Bowen KF. The effects of delayed and displaced visual feedback on motor control. J Mot Behav. 1980;12(2):91-101. doi: 10.1080/00222895.1980.10735209 pmid: 15215054

13. Raud L, Westerhausen R, Dooley N, Huster RJ. Differences in unity: The go/no-go and stop signal tasks rely on different mechanisms. Neuroimage. 2020;210:116582. doi: 10.1016/j.neuroimage.2020.116582 pmid: 31987997

14. Schultze-Kraft M, Birman D, Rusconi M, Allefeld C, Gorgen K, Dahne S, et al. The point of no return in vetoing self-initiated movements. Proc Natl Acad Sci U S A. 2016;113(4):1080-1085. doi: 10.1073/pnas. 1513569112 pmid: 26668390

15.Zelaznik HN, Rosenbaum DA. Timing processes are correlated when tasks share a salient event. $J$ Exp Psychol Hum Percept Perform. 2010;36(6):1565-1575. doi: 10.1037/a0020380 pmid: 20731516

16. Raud L, Huster RJ, Ivry RB, Labruna L, Messel MS, Greenhouse I. A single mechanism for global and selective response inhibition under the influence of motor preparation. BioRxiv. 2020;3(16):993261. doi: 10.1101/2020.03.16.993261

17.Xu KZ, Mayse JD, Courtney S. Evidence for selective adjustments of inhibitory control in a variant of the stop signal task. $Q J$ Exp Psychol (Hove). 2019;72(4):818-831. doi: 10.1177/1747021818768721 pmid: 29589791

18. Nguyen AT, Albrecht MA, Lipp OV, Marinovic W. Motor Output Matters: Evidence of a Continuous Relationship Between Stop/No-go P300 Amplitude and Response Force on Failed Inhibitions at the Trial-Level. BioRxiv. 2019:706978. doi: 10.1101/706978

19. Quílez Maimón A, Rojas Ruiz F. Assessment of the point of no return in choice reaction time under uncertainty conditions in basketball pass.2017.

20. Gao L, Zelaznik HN. The modification of an already-programmed response: a new interpretation of Henry and Harrison (1961). J Mot Behav. 1991;23(3):221-223. doi: 10.1080/00222895.1991.10118365 pmid: 14766519

21. Mele A. Free Will and Neuroscience: Decision Times and the Point of No Return:: Brill, Rodopi; 2019. 
22. Sperl L, Canal-Bruland R. Interindividual differences in the capability to change automatized movement patterns. Acta Psychol (Amst). 2020;204:103027. doi: 10.1016/j.actpsy.2020.103027 pmid: 32050121

23. Dupuis A, Indralingam M, Chevrier A, Crosbie J, Arnold P, Burton CL, et al. Response Time Adjustment in the Stop Signal Task: Development in Children and Adolescents. Child Dev. 2019;90(2):e263-e272. doi: 10.1111/cdev.13062 pmid: 29603204

24.Friedman NP, Miyake A, Young SE, DeFries JC, Corley RP, Hewitt JK. Individual differences in executive functions are almost entirely genetic in origin. $J$ Exp Psychol Gen. 2008;137(2):201-225. doi: 10.1037/0096-3445.137.2.201 pmid: 18473654

25. Coxon JP, Van Impe A, Wenderoth N, Swinnen SP. Aging and inhibitory control of action: corticosubthalamic connection strength predicts stopping performance. J Neurosci. 2012;32(24):8401-8412. doi: 10.1523/JNEUROSCI.6360-11.2012 pmid: 22699920

26. Alouche SR, Sant'Anna GN, Biagioni G, Ribeiro-do-Valle LE. Influence of cueing on the preparation and execution of untrained and trained complex motor responses. Braz J Med Biol Res. 2012;45(5):425435. doi: 10.1590/s0100-879x2012007500053 pmid: 22473319

27. Aron AR, Fletcher PC, Bullmore ET, Sahakian BJ, Robbins TW. Stop-signal inhibition disrupted by damage to right inferior frontal gyrus in humans. Nat Neurosci. 2003;6(2):115-116. doi: 10.1038/nn1003 pmid: 12536210

28. Drummond NM, Cressman EK, Carlsen AN. Go-activation endures following the presentation of a stopsignal: evidence from startle. J Neurophysiol. 2017;117(1):403-411. doi: 10.1152/jn.00567.2016 pmid: 27832599

29. Boehler CN, Appelbaum LG, Krebs RM, Chen LC, Woldorff MG. The role of stimulus salience and attentional capture across the neural hierarchy in a stop-signal task. PLoS One. 2011;6(10):e26386. doi: 10.1371/journal.pone.0026386 pmid: 22022611

30. Shelton J, Kumar GP. Comparison between auditory and visual simple reaction times. Neurosci Med. 2010;1(1):30-32. doi: 10.4236/nm.2010.11004

31. Magill RA, Anderson DI. Motor learning and control: Concepts and applications: McGraw-Hill New York; 2007.

32. Schwartz M, Manickum O. Programming Arduino with LabVIEW: Packt Publishing Ltd2015.

33. de Jong R, Coles MGH, Logan GD, Gratton G. In search of the point of no return: the control of response processes. J Exp Psychol Hum Percept Perform. 1990;16(1):164-182. doi: 10.1037/0096-1523.16.1.164 pmid: 2137517

34.Jiang Y, Li Z, Zhao Y, Xiao X, Zhang W, Sun P, et al. Targeting brain functions from the scalp: Transcranial brain atlas based on large-scale fMRI data synthesis. Neuroimage. 2020;210:116550. doi: 10.1016/j.neuroimage.2020.116550 pmid: 31981781

35. Carter JD, Farrow M, Silberstein RB, Stough C, Tucker A, Pipingas A. Assessing inhibitory control: a revised approach to the stop signal task. J Atten Disord. 2003;6(4):153-161. doi: 10.1177/108705470300600402 pmid: 12931073

36. Verbruggen F, Logan GD. Models of response inhibition in the stop-signal and stop-change paradigms. Neurosci Biobehav Rev. 2009;33(5):647-661. doi: 10.1016/j.neubiorev.2008.08.014 pmid: 18822313

37. Boucher L, Palmeri TJ, Logan GD, Schall JD. Inhibitory control in mind and brain: an interactive race model of countermanding saccades. Psychol Rev. 2007;114(2):376-397. doi: 10.1037/0033295X.114.2.376 pmid: 17500631

38. Bryce D, Bratzke D. Introspective reports of reaction times in dual-tasks reflect experienced difficulty rather than timing of cognitive processes. Conscious Cogn. 2014;27:254-267. doi: 10.1016/j.concog.2014.05.011 pmid: 24956469 